\title{
Solution of fuzzy singular integral equation with Abel's type kernel using a novel hybrid method
}

\author{
S. Bushnaq ${ }^{1}$, Z. Ullah², A. Ullah² and K. Shah ${ }^{2 *}$
}

\section{"Correspondence:}

kamalshah408@gmail.com

${ }^{2}$ Department of Mathematics,

University of Malakand, Dir(L),

Khyber Pakhtunkhwa, Pakistan

Full list of author information is

available at the end of the article

\section{Springer}

\begin{abstract}
In this paper, we are applying a novel analytical hybrid method to find the solution of a fuzzy Volterra Abel's integral equation of the second kind. The fuzzy number is used in its parametric form under which the fuzzy Volterra Abel's integral equation will be converted into a system of integral equations as in a crisp case. Moreover, to solve the general fuzzy Volterra integral equation with Abel's type kernel, and to show that the proposed method is efficient, a few accurate and simple examples are given for the demonstration of our results.
\end{abstract}

MSC: Primary 45Exx; 45E10; secondary 47B35

Keywords: Laplace transform; Adomian Decomposition Method; Fuzzy Volterra Abel's integral equation; Laplace Adomian Decomposition Method; Fuzzy number

\section{Introduction}

There are many fields in real life which face uncertainty in data, like environmental sciences, medical sciences, economics, social sciences, and so on. To study these problems, the fuzzy set theory is one of the most important theories introduced by Zadeh in 1965 [1]. The fuzzy set theory has been used in various other fields, i.e., fuzzy fixed-point theory, fuzzy topology, fuzzy control systems, fuzzy automata, etc. Chang and Zadeh further extended the concept of a fuzzy set and introduced fuzzy mapping and control [2]. On the basis of fuzzy mapping and control, many researchers generalized this concept to introduce elementary fuzzy calculus [3-6]. Fuzzy logic has many applications in daily life, like vacuum cleaner, washing machine, robotics, stock trading, etc. [7]. The contrast enhancement algorithm based on fuzzy contextual information of the images is discussed by Parihar et al. in [8]. In the last few decades, fuzzy integral equations received considerable interest in studying the field of physical sciences. Dobius and Prade were among those who introduced the basic idea of fuzzy integral equations [6]. Further, extensions of fuzzy integral equations were made by Matloka and Nanda $[9,10]$. In applied sciences, fuzzy integral equations have vast applications in geography, relational physics, biology, medical sciences and many more. However, to deal with such types of problems, where information is vague and imprecise rather than crisp, the parameters are represented by

(c) The Author(s) 2020. This article is licensed under a Creative Commons Attribution 4.0 International License, which permits use, sharing, adaptation, distribution and reproduction in any medium or format, as long as you give appropriate credit to the original author(s) and the source, provide a link to the Creative Commons licence, and indicate if changes were made. The images or other third party material in this article are included in the article's Creative Commons licence, unless indicated otherwise in a credit line to the material. If material is not included in the article's Creative Commons licence and your intended use is not permitted by statutory regulation or exceeds the permitted use, you will need to obtain permission directly from the copyright holder. To view a copy of this licence, visit http://creativecommons.org/licenses/by/4.0/. 
fuzzy numbers. Such problems can be modeled using fuzzy integral equations. Therefore, many researchers worked on these models to investigate the solutions of fuzzy integral equations.

Recently, many analytical and numerical approaches were proposed to solve fuzzy integral and differential equations, for example, Jafarian and Nia applied Taylor expansion method for the system of fuzzy Volterra integral equations [11]. Ghanbari and Allahviranloo, and also Matinfar and Saeidy, proposed the Homotopy Perturbation Method (HPM) and Homotopy Analysis Method (HAM) for solving fuzzy integral equations [12, 13]. For the solution of fuzzy Fredholm integral equations of the second kind, Ezzati and Ziari discussed an approach which is based on Bernstein polynomials [14]. Similarly to generalized differentiability of fuzzy differential equations, hybrid retarded delay differential equations were solved numerically by Runge-Kutta method $[15,16]$. For noninteger order, the interval differential equations were solved in [17]. Numerical simulation of two viscoelastic systems based on differential equations of noninteger order under uncertainty were studied in [18]. The authors investigate fuzzy fractional differential equations and optimal control of nonlocal evolution equations in [19]. Fuzzy linear integral equations were further generalized to nonlinear fuzzy integral equations [20]. Shafiee et al. proposed a numerical algorithm based on the predictor-corrector method for nonlinear fuzzy integral equations [21]. The existence and uniqueness of solutions of fuzzy integral equations, especially, the solutions of fuzzy Volterra integro-differential equations of the second kind with a fuzzy kernel and linear Fredholm fuzzy integral equations of the second kind in artificial neural networks, were established by many researchers [22-25].

During the last decades, the subject of fractional calculus, and its potential applications, have gained increasing importance, mainly because it has become a powerful tool with accurate and successful results in modeling several complex phenomena in numerous seemingly diverse and widespread fields of science and engineering [26-29]. Fractional calculus is not only a productive and emerging field, it also represents a new philosophy how to construct and apply a certain type of nonlocal operators to real world problems. Those possessing both nonlocal effects as well as uncertainty behaviors represent interesting phenomena. Abel's singular integral equation is a particular type of singular integral equation where in the range of integration the kernel has a singularity. Abel investigated such equations for the first time during his study of mathematical physics [30, 31].

Abel's singular integral equations occur in various areas of scientific fields, such as seismology, microscopy, radio astronomy, electron emission, radar ranging, atomic scattering, plasma diagnostics, optical fiber evaluation, and X-ray radiography [32, 33]. In the biomedical branch, Abel's equations are mostly used in X-ray CT (computerized tomography), which has revolutionized diagnostic radiology since its introduction [34]. Abel's type kernel integral equations are very important for several models in solid mechanics, physics, and applied sciences which rely on this type of integral equation.

Thus, there are many techniques already in the literature which are a little bit complicated and in the solution an error term is produced. This motivates us to solve Abel's singular integral equation by an alternative method in order to overcome such difficulties. Therefore, the method of LADM is used to find the solution with more accuracy and to reduce the error term as much as possible.

In HAM the convergence of a series solution depends upon four factors, i.e., the initial guess, auxiliary linear operator, auxiliary function which we define for homotopy, and 
auxiliary parameter $\hbar$. Further, if we select $\hbar=-1$, and the auxiliary function also equal to 1, we get HPM. Hence HPM is a spacial case of HAM whose convergence [35] is only dependent upon two factors, the auxiliary linear operator and the initial guess. So, given the initial guess and the auxiliary linear operator, HPM approach cannot provide other ways to ensure that the solution is convergent. On the other hand, LADM solutions for both linear and nonlinear problems are obtained in series form showing higher convergence order of the method. Among all other analytical methods, LADM is an efficient analytical method to solve nonlinear problems with differential or integral equations. This is a hybrid method form employing a combination of two powerful methods, Laplace transform and Adomian decomposition method. The mentioned method does not need any discretization or linearization. It also does not need a predefined parameter as in HAM which controls this method. Therefore LADM [36] is considered as an efficient analytical technique for treating those equations that represent nonlinear models. Here we remark that LADM without initial condition converges towards a particular solution [37].

This paper is organized as follows: In Sect. 2, some basic results and definitions are presented. In Sect. 3, we establish a scheme for solving a general fuzzy Volterra singular integral equation with Able's type kernel. Several examples are given in Sect. 4, and conclusions are drawn in Sect. 5.

\section{Preliminaries}

We recall some definitions needed through the paper.

Definition 1 ([38]) Let $f(x)$ be acontinuous fuzzy-valued function and suppose $e^{-p x} \odot f(x)$ is improperly fuzzy Riemann integrable on $[0, \infty)$, then $\int_{0}^{\infty} e^{-p x} \odot f(x) d x$ is said to be a fuzzy Laplace transform, denoted as

$$
L[f(x)]=\int_{0}^{\infty} e^{-p x} \odot f(x) d x \quad(p>0 \text { and is an integer }) .
$$

Definition 2 ([31]) Adomian Decomposition Method is defined for the unknown function $u(x, t)$ by an infinite series as $u(x, t)=\sum_{i=0}^{\infty} u_{i}(x, t)$ where the components $u_{i}(x, t)$ will be determined recursively.

Definition 3 ([39]) Let $g_{1}$ and $g_{2}$ be two fuzzy-valued functions. Then the fuzzy convolution is defined for $x>0$ by

$$
\left(g_{1} * g_{2}\right)(x)=\int_{0}^{x} g_{1}(\tau) \cdot g_{2}(x-\tau) d \tau
$$

which exists if $g_{1}$ and $g_{2}$ are, say, piecewise continuous. Putting $v=x-\tau$ gives

$$
\left(g_{1} * g_{2}\right)(x)=\int_{0}^{x} g_{2}(v) \cdot g_{1}(x-v) d v=\left(g_{2} * g_{1}\right)(x),
$$

that is, the fuzzy convolution is commutative.

Definition 4 ([40]) The Mittag-Leffler function $E_{\alpha}(z)$ with one parameter is defined as

$$
E_{\alpha}(z)=\sum_{n=0}^{\infty} \frac{z^{n}}{\Gamma(\alpha n+1)}
$$


where $\alpha>0, z$ is a complex number, and $\Gamma$ is the gamma function which is defined for $\operatorname{Re} z>0$ as

$$
\Gamma(z)=\int_{0}^{\infty} x^{z-1} e^{x} d x
$$

Definition 5 ([4]) A mapping $v: \mathbb{R} \rightarrow[0,1]$ is said to be a fuzzy number if the following conditions are satisfied:

(i) $v$ is upper semi-continuous;

(ii) $v\left\{\mu\left(y_{1}\right)+\mu\left(y_{2}\right)\right\} \geq \min \left\{v\left(y_{1}\right), v\left(y_{2}\right)\right\}$;

(iii) there exist $y_{0} \in \mathbb{R}$ such that $v\left(y_{0}\right)=1$, i.e., $v$ is normal;

(iv) $c l\{y \in \mathbb{R}, v(y)>0\}$ is compact.

Here we denote the set of all fuzzy numbers by $E$.

Definition 6 ([41]) The parametric form of a fuzzy number can be written in the form of an ordered pair as $(\underline{v}(\kappa), \bar{v}(\kappa))$ such that $0 \leq \kappa \leq 1$ and the following conditions hold:

(i) $\underline{v}(\kappa)$ is a bounded left-continuous nondecreasing function on $[0,1]$;

(ii) $\bar{v}(\kappa)$ is bounded right-continuous nonincreasing function on [0,1];

(iii) $\underline{v}(\kappa) \leq \bar{v}(\kappa)$.

Also $\kappa$ is called a crisp number if $\underline{v}(\kappa)=\bar{v}(\kappa)=\kappa$.

Definition 7 ([42]) Let $\widetilde{\phi}:\left[\theta_{1}, \theta_{2}\right] \rightarrow E$ be a fuzzy-valued function, for every partition $Q=\left\{y_{0}, y_{1}, \ldots, y_{m}\right\}$ of $\left[\theta_{1}, \theta_{2}\right]$, and for every $\eta_{\hbar} \in\left[y_{j-1}, y_{j}\right], 1 \leq \hbar \leq m$, we define

$$
S_{q}=\sum_{j=0}^{m} \widetilde{\phi}\left(\eta_{\hbar}\right)\left(y_{j}-y_{j-1}\right) .
$$

Now let $\triangle_{j}=\max \left|y_{j}-y_{j-1}\right|, j=1,2, \ldots, m$, then for $\widetilde{\phi}(y)$, the definite integral over $\left[\theta_{1}, \theta_{2}\right]$ can be written as

$$
\lim _{\Delta j \rightarrow 0} S_{q}=\int_{\theta_{1}}^{\theta_{2}} \widetilde{\phi}(y) d y
$$

and the parametric form can be written as

$$
\int_{\theta_{1}}^{\theta_{2}} \widetilde{\phi}(y) d y=\left(\int_{\theta_{1}}^{\theta_{2}} \widetilde{\phi}(y, \kappa) d y, \int_{\theta_{1}}^{\theta_{2}} \widetilde{\phi}(y, \kappa) d y\right) .
$$

Theorem 1 (Fuzzy convolution theorem, [39]) Let $\psi$ and $\phi$ be fuzzy-valued functions on $[0, \infty)$, where $\psi$ and $\phi$ are piecewise continuous and of exponential order $q$, then

$$
L[(\psi * \phi)(x)]=L[\psi(x)] \cdot L[\phi(x)],
$$

where $L$ represents the Laplace transform.

\section{Fuzzy Volterra singular integral equation with Abel's type kernel}

The standard form of a Volterra linear integral equation, where the integration has a variable limit rather than a constant, is of the form

$$
\rho(y) \psi(y)=\phi(y)+\lambda \int_{0}^{y} K(y, t) \psi(t) d t,
$$


where $\phi(y), k(y, t)$ are known functions and $\psi(y)$ is an unknown to be determined and enters linearly. If $\rho(y)=0$ then Eq. (7) is said to be a Volterra integral equation of the first kind, and when $\rho(y)=1$ then Eq. (7) is said to be a Volterra integral equation of the second kind and has been studied widely in [31].

Here we discuss the singular fuzzy Volterra linear integral equation with Abel's type kernel which can be written in a general form as

$$
\psi(y)=\phi(y)+\int_{0}^{y} \frac{\psi(t)}{\sqrt{y-t}} d t
$$

where $\phi(y)$ is a fuzzy-valued function which is predefined on the interval $\left[\theta_{1}, \theta_{2}\right]$ and we seek to find a fuzzy solution $\psi(y)$, which is unknown, and $(y-t)^{-1 / 2}$ is said to be the kernel of Abel's integral equation. We can rewrite Eq. (8) as follows:

$$
\left\{\begin{array}{l}
\underline{\psi}(y, \kappa)=\underline{\phi}(y, \kappa)+\int_{0}^{y} \frac{\underline{\psi}(t, \kappa)}{\sqrt{y-t}} d t \\
\bar{\psi}(y, \kappa)=\bar{\phi}(y, \kappa)+\int_{0}^{y} \frac{\bar{\psi}(t, \kappa)}{\sqrt{y-t}} d t
\end{array}\right.
$$

where $\kappa, y \in[0,1]$.

In the following, we provide a basic idea of LADM to solve Eq. (8) in its parametric form given in Eq. (9).

To solve Eq. (8), applying Laplace transform to both sides of Eq. (9), we have

$$
\left\{\begin{array}{l}
L[\underline{\psi}(y, \kappa)]=L[\underline{\phi}(y, \kappa)]+L\left[\int_{0}^{y} \frac{\underline{\psi}(t, \kappa)}{\sqrt{y-t}} d t\right], \\
L[\bar{\psi}(y, \kappa)]=L[\bar{\phi}(y, \kappa)]+L\left[\int_{0}^{y} \frac{\bar{\psi}(t, \kappa)}{\sqrt{y-t}} d t\right] .
\end{array}\right.
$$

In view of Theorem 1, Eq. (10) yields

$$
\begin{gathered}
\left\{\begin{array}{l}
L[\underline{\psi}(y, \kappa)]=L[\phi(y, \kappa)]+L\left[y^{-1 / 2}\right] \cdot L[\underline{\psi}(y, \kappa)], \\
L[\bar{\psi}(y, \kappa)]=L[\bar{\phi}(y, \kappa)]+L\left[y^{-1 / 2}\right] \cdot L[\bar{\psi}(y, \kappa)],
\end{array}\right. \\
\left\{\begin{array}{l}
L[\bar{\psi}(y, \kappa)]=L[\phi(y, \kappa)]+\sqrt{\frac{\pi}{t}} L[\underline{\psi}(y, \kappa)], \\
L[\bar{\psi}(y, \kappa)]=L[\bar{\phi}(y, \kappa)]+\sqrt{\frac{\pi}{t}} L[\bar{\psi}(y, \kappa)] .
\end{array}\right.
\end{gathered}
$$

Upon using inverse the Laplace transform on both sides of Eq. (11), we obtain

$$
\left\{\begin{array}{l}
\psi(y, \kappa)=\underline{\phi}(y, \kappa)+L^{-1}\left[\sqrt{\frac{\pi}{t}} L[\underline{\psi}(y, \kappa)]\right], \\
\overline{\bar{\psi}}(y, \kappa)=\bar{\phi}(y, \kappa)+L^{-1}\left[\sqrt{\frac{\pi}{t}} L[\overline{\bar{\psi}}(y, \kappa)]\right] .
\end{array}\right.
$$

Let the solution of Eq. (12) be in the form of an infinite series as

$$
\left\{\begin{array}{l}
\psi(y, \kappa)=\sum_{j=0}^{\infty} \underline{u}_{j}(y, \kappa), \\
\bar{\psi}(y, \kappa)=\sum_{j=0}^{\infty} \bar{u}_{j}(y, \kappa),
\end{array}\right.
$$

where $\left(\underline{u}_{j}, \bar{u}_{j}\right), \forall j=0,1,2, \ldots$ are unknown functions to be determined. 
Putting Eq. (13) into Eq. (12), we have

$$
\left\{\begin{array}{l}
\sum_{j=0}^{\infty} \underline{u}_{j}(y, \kappa)=\underline{\phi}(y, \kappa)+L^{-1}\left[\sqrt{\frac{\pi}{t}} L\left[\sum_{j=0}^{\infty} \underline{u}_{j}(y, \kappa)\right]\right] \\
\sum_{j=0}^{\infty} \bar{u}_{j}(y, \kappa)=\bar{\phi}(y, \kappa)+L^{-1}\left[\sqrt{\frac{\pi}{t}} L\left[\sum_{j=0}^{\infty} \bar{u}_{j}(y, \kappa)\right]\right] .
\end{array}\right.
$$

Comparing termwise both sides of Eq. (14), we get

$$
\begin{aligned}
& \left\{\begin{array}{l}
\underline{u}_{0}(y, \kappa)=\underline{\phi}(y, \kappa), \\
\bar{u}_{0}(y, \kappa)=\bar{\phi}(y, \kappa),
\end{array}\right. \\
& \left\{\begin{array}{l}
\underline{u}_{1}(y, \kappa)=L^{-1}\left[\sqrt{\frac{\pi}{t}} L\left[\underline{u}_{0}(y, \kappa)\right]\right], \\
\bar{u}_{1}(y, \kappa)=L^{-1}\left[\sqrt{\frac{\pi}{t}} L\left[\bar{u}_{0}(y, \kappa)\right]\right],
\end{array}\right. \\
& \vdots \\
& \left\{\begin{array}{l}
\underline{u}_{n+1}(y, \kappa)=L^{-1}\left[\sqrt{\frac{\pi}{t}} L\left[\underline{u}_{n}(y, \kappa)\right]\right], \\
\bar{u}_{n+1}(y, \kappa)=L^{-1}\left[\sqrt{\frac{\pi}{t}} L\left[\bar{u}_{n}(y, \kappa)\right]\right],
\end{array}\right.
\end{aligned}
$$

where $n \geq 0$. Putting all the values of lower and upper term solution into Eq. (13), we get the solution of the fuzzy Abel's singular integral equation in Eq. (8) in parametric form.

In the above iterative scheme the initial guess $u_{0}(y, \kappa)$ is very important since the initial guess either gives the exact solution directly by producing noise terms or gives the solution in closed form which shows the simplicity and accuracy of the said scheme.

\section{Numerical examples}

A few examples are solved in this section by applying the proposed method to show the simplicity, accuracy of the method, and why the given scheme is more applicable.

Example 1 ([43]) Let the fuzzy singular Volterra integral equation of the second kind with Abel's type kernel in parametric form be given by

$$
\left\{\begin{array}{l}
\psi(y, \kappa)=\underline{\phi}(y, \kappa)+\int_{0}^{y} \frac{\underline{\psi}(t, \kappa)}{\sqrt{y-t}} d t \\
\bar{\psi}(y, \kappa)=\bar{\phi}(y, \kappa)+\int_{0}^{y} \frac{\frac{\bar{\psi}(t, \kappa)}{\sqrt{y-t}} d t}{}
\end{array}\right.
$$

where the lower and upper nonhomogenous terms are

$$
\left\{\begin{array}{l}
\underline{\phi}(y, \kappa)=\frac{\kappa}{15}\left(15 y^{2}+16 y^{5 / 2}\right) \\
\bar{\phi}(y, \kappa)=\frac{1}{15}\left((2-\kappa) y^{2}-(32-16 \kappa) y^{5 / 2}\right)
\end{array}\right.
$$

and the exact solution is $\left(\kappa y^{2},(2-\kappa) y^{2}\right)$.

To solve the given example using LADM proceed as follows:

First, we solve for the lower limit solution of Eq. (16) such that

$$
\underline{\psi}(y, \kappa)=\underline{\phi}(y, \kappa)-\int_{0}^{y} \frac{\underline{\psi}(t, \kappa)}{\sqrt{y-t}} d t
$$


where

$$
\phi(y, \kappa)=\frac{\kappa}{15}\left(15 y^{2}+16 y^{5 / 2}\right) .
$$

Applying Laplace transform and Theorem 1 and then using the inverse Laplace transform on Eq. (17), we have

$$
\underline{\psi}(y, \kappa)=\underline{\phi}(y, \kappa)-L^{-1}\left[\sqrt{\frac{\pi}{t}} L[\underline{\psi}(y, \kappa)]\right],
$$

the solution of Eq. (18) will be in the form of an infinite series. So let

$$
\underline{\psi}(y, \kappa)=\sum_{j=o}^{\infty} \underline{u}_{j}(y, \kappa) .
$$

Putting Eq. (19) in Eq. (18), we get

$$
\begin{aligned}
& \sum_{j=o}^{\infty} \underline{u}_{j}(y, \kappa)=\underline{\phi}(y, \kappa)-L^{-1}\left[\sqrt{\frac{\pi}{t}} L\left[\sum_{j=0}^{\infty} \underline{u}_{j}(y, \kappa)\right]\right], \\
& \underline{u}_{0}(y, \kappa)+\underline{u}_{1}(y, \kappa)+\cdots=\frac{\kappa}{15}\left(15 y^{2}+16 y^{5 / 2}\right)-L^{-1}\left[\sqrt{\frac{\pi}{t}} L\left[\underline{u}_{0}(y, \kappa)\right]\right]-\cdots .
\end{aligned}
$$

Comparing Eq. (20) termwise and then solving, we get

$$
\left\{\begin{array}{l}
\underline{u}_{o}(y, \kappa)=\frac{\kappa}{15}\left(15 y^{2}+16 y^{5 / 2}\right), \\
\underline{u}_{1}(y, \kappa)=-L^{-1}\left[\sqrt{\frac{\pi}{t}} L\left[\underline{u}_{0}(y, \kappa)\right]\right]=-\frac{\kappa}{15}\left[16 y^{5 / 2}+5 \pi y^{3}\right], \\
\underline{u}_{2}(y, \kappa)=L^{-1}\left[\sqrt{\frac{\pi}{t}} L\left[\underline{u}_{1}(y, \kappa)\right]\right]=\frac{\kappa}{15}\left[5 \pi y^{3}+\frac{32}{7} \pi y^{7 / 2}\right], \\
\underline{u}_{3}(y, \kappa)=-L^{-1}\left[\sqrt{\frac{\pi}{t}} L\left[\underline{u}_{2}(y, \kappa)\right]\right]=-\frac{\kappa}{15}\left[\frac{32}{7} \pi y^{7 / 2}+\frac{5}{4} \pi^{2} y^{4}\right], \\
\underline{u}_{4}(y, \kappa)=-L^{-1}\left[\sqrt{\frac{\pi}{t}} L\left[\underline{u}_{3}(y, \kappa)\right]\right]=\frac{\kappa}{15}\left[\frac{5}{4} \pi^{2} y^{4}+\frac{64}{63} \pi^{2} y^{9 / 2}\right], \\
\underline{u}_{5}(y, \kappa)=-L^{-1}\left[\sqrt{\frac{\pi}{t}} L\left[\underline{u}_{4}(y, \kappa)\right]\right]=-\frac{\kappa}{15}\left[\frac{64}{63} \pi^{2} y^{9 / 2}+\frac{1}{4} \pi^{3} y^{5}\right], \\
\vdots
\end{array}\right.
$$

then putting Eq. (21) termwise into Eq. (19) on the right-hand side, which produces noise term, by cancelation we get

$$
\underline{\psi}(y, \kappa)=\kappa y^{2} .
$$

Now for the upper limit solution, applying Laplace transform, Theorem 1, and the inverse Laplace transform on upper limit equation in Eq. (16), we get

$$
\bar{\psi}(y, \kappa)=\bar{\phi}(y, \kappa)-L^{-1}\left[\sqrt{\frac{\pi}{t}} L[\bar{\psi}(y, \kappa)]\right] .
$$

Let the solution of Eq. (22) be in the form of an infinite series as

$$
\bar{\psi}(y, \kappa)=\sum_{j=0}^{\infty} \bar{u}_{j}(y, \kappa) .
$$


Putting Eq. (23) in Eq. (22), we have

$$
\begin{aligned}
& \sum_{j=o}^{\infty} \bar{u}_{j}(y, \kappa)=\bar{\phi}(y, \kappa)-L^{-1}\left[\sqrt{\frac{\pi}{t}} L\left[\sum_{j=0}^{\infty} \bar{u}_{j}(y, \kappa)\right]\right] \\
& \bar{u}_{0}(y, \kappa)+\bar{u}_{1}(y, \kappa)+\cdots \\
& \quad=\frac{1}{15}\left((2-\kappa) y^{2}-(32-16 \kappa) y^{5 / 2}\right)-L^{-1}\left[\sqrt{\frac{\pi}{t}} L\left[\bar{u}_{0}(y, \kappa)\right]\right]-\cdots
\end{aligned}
$$

Comparing Eq. (24) termwise and then solving, we get

$$
\left\{\begin{array}{l}
\bar{u}_{0}(y, \kappa)=\frac{1}{15}\left((2-\kappa) y^{2}-(32-16 \kappa) y^{5 / 2}\right), \\
\bar{u}_{1}(y, \kappa)=-L^{-1}\left[\sqrt{\frac{\pi}{t}} L\left[\bar{u}_{0}(y, \kappa)\right]\right]=-(2-\kappa)\left[\frac{16}{15} y^{5 / 2}+\frac{1}{3} \pi y^{3}\right], \\
\bar{u}_{2}(y, \kappa)=-L^{-1}\left[\sqrt{\frac{\pi}{t}} L\left[\bar{u}_{1}(y, \kappa)\right]\right]=(2-\kappa)\left[\frac{1}{3} \pi y^{3}+\frac{32}{105} \pi y^{7 / 2}\right], \\
\bar{u}_{3}(y, \kappa)=-L^{-1}\left[\sqrt{\frac{\pi}{t}} L\left[\bar{u}_{2}(y, \kappa)\right]\right]=-(2-\kappa)\left[\frac{32}{105} \pi y^{7 / 2}+\frac{1}{12} \pi^{2} y^{4}\right], \\
\bar{u}_{4}(y, \kappa)=-L^{-1}\left[\sqrt{\frac{\pi}{t}} L\left[\bar{u}_{3}(y, \kappa)\right]\right]=(2-\kappa)\left[\frac{1}{12} \pi^{2} y^{4}+\frac{64}{945} \pi^{2} y^{9 / 2}\right], \\
\bar{u}_{5}(y, \kappa)=-L^{-1}\left[\sqrt{\frac{\pi}{t}} L\left[\bar{u}_{4}(y, \kappa)\right]\right]=-(2-\kappa)\left[\frac{64}{945} \pi^{2} y^{9 / 2}+\frac{1}{60} \pi^{3} y^{5}\right], \\
\vdots
\end{array}\right.
$$

then putting Eq. (25) termwise into Eq. (23) on the right-hand side and canceling the noise terms, we get

$$
\bar{\psi}(y, \kappa)=(2-\kappa) y^{2} .
$$

Thus the lower and upper limit solution of Eq. (16) is

$$
(\underline{\psi}(y, \kappa), \bar{\psi}(y, \kappa))=\left(\kappa y^{2},(2-\kappa) y^{2}\right)
$$

which is the exact solution. Here we remark that the solution we obtain via LADM is in complete agreement with the solution in [34] by HPM and modified HPM. Here we claim that LADM is simple and needs no extra parameter on which the convergence of the solution depends.

Example 2 ([43]) Consider the fuzzy singular integral equation with Abel's type kernel in parametric form given by

$$
\left\{\begin{array}{l}
\underline{\psi}(y, \kappa)=\left(y+\frac{4}{3} y^{3 / 2}\right)(4+\kappa)-\int_{0}^{y} \frac{f(t, \kappa)}{\sqrt{y-t}} d t, \\
\bar{\psi}(y, \kappa)=\left(y+\frac{4}{3} y^{5 / 2}\right)(6-\kappa)-\int_{0}^{y} \frac{\underline{\psi}(t, \kappa)}{\sqrt{y-t}} d t
\end{array}\right.
$$

where the exact solution is

$$
((4+\kappa) y,(6-\kappa) y) .
$$


Applying Laplace transform, Theorem 1, and the inverse Laplace transform of Eq. (26) to the lower limit equation, we get

$$
\underline{\psi}(y, \kappa)=\left(y+\frac{4}{3} y^{3 / 2}\right)(4+\kappa)-L^{-1}\left[\sqrt{\frac{\pi}{t}} L[\underline{\psi}(y, \kappa)]\right] .
$$

Consider the lower limit solution of Eq. (27) in the form of an infinite series as

$$
\underline{\psi}(y, \kappa)=\sum_{j=0}^{\infty} \underline{u}_{j}(y, \kappa)
$$

Putting Eq. (28) into Eq. (27), we have

$$
\sum_{j=0}^{\infty} \underline{u}_{j}(y, \kappa)=\left(y+\frac{4}{3} y^{3 / 2}\right)(4+\kappa)-L^{-1}\left[\sqrt{\frac{\pi}{t}} L\left[\sum_{j=0}^{\infty} \underline{u}_{j}(y, \kappa)\right]\right] .
$$

Comparing Eq. (29) termwise and solving, we get

$$
\left\{\begin{array}{l}
\underline{u}_{0}(y, \kappa)=(4+\kappa)\left[y+\frac{4}{3} y^{3 / 2}\right], \\
\underline{u}_{1}(y, \kappa)=-L^{-1}\left[\sqrt{\frac{\pi}{t}} L\left[\underline{u}_{0}(y, \kappa)\right]\right]=-(4+\kappa)\left[\frac{4}{3} y^{3 / 2}+\frac{1}{2} \pi y^{2}\right], \\
\underline{u}_{2}(y, \kappa)=-L^{-1}\left[\sqrt{\frac{\pi}{t}} L\left[\underline{u}_{1}(y, \kappa)\right]\right]=(4+\kappa)\left[\frac{1}{2} \pi y^{2}+\frac{8}{15} \pi y^{5 / 2}\right], \\
\underline{u}_{3}(y, \kappa)=-L^{-1}\left[\sqrt{\frac{\pi}{t}} L\left[\underline{u}_{2}(y, \kappa)\right]\right]=-(4+\kappa)\left[\frac{8}{15} \pi y^{5 / 2}+\frac{1}{6} \pi^{2} y^{3}\right], \\
\vdots
\end{array}\right.
$$

then putting all the values of Eq. (30) termwise into Eq. (28) on the right-hand side and simplifying, we obtain

$$
\underline{\psi}(y, \kappa)=(4+\kappa) y .
$$

Then for the upper limit solution, take the upper limit equation of Eq. (26) and have

$$
\bar{\psi}(y, \kappa)=\left(y+\frac{4}{3} y^{5 / 2}\right)(6-\kappa)-\int_{0}^{y} \frac{\underline{\psi}(t, \kappa)}{\sqrt{y-t}} d t .
$$

Applying Laplace transform, Theorem 1, and the inverse Laplace transform, we get

$$
\bar{\psi}(y, \kappa)=\left(y+\frac{4}{3} y^{5 / 2}\right)(6-\kappa)-L^{-1}\left[\sqrt{\frac{\pi}{t}} L[\bar{\psi}(y, \kappa)]\right] .
$$

Let the solution of Eq. (32) be in the form of an infinite series as

$$
\underline{\psi}(y, \kappa)=\sum_{j=0}^{\infty} \underline{u}_{j}(y, \kappa)
$$

Putting Eq. (33) into Eq. (32) gives

$$
\sum_{j=0}^{\infty} \bar{u}_{j}(y, \kappa)=\left(y+\frac{4}{3} y^{5 / 2}\right)(6-\kappa)-L^{-1}\left[\sqrt{\frac{\pi}{t}} L\left[\sum_{j=0}^{\infty} \bar{u}_{j}(y, \kappa)\right]\right] .
$$


Now comparing Eq. (34) termwise and solving, we obtain

$$
\left\{\begin{array}{l}
\bar{u}_{0}(y, \kappa)=(6-\kappa)\left[y+\frac{4}{3} y^{5 / 2}\right], \\
\bar{u}_{1}(y, \kappa)=-L^{-1}\left[\sqrt{\frac{\pi}{t}} L\left[\bar{u}_{0}(y, \kappa)\right]\right]=-(6-\kappa)\left[\frac{4}{3} y^{3 / 2}+\frac{1}{2} \pi y^{2}\right] \\
\bar{u}_{2}(y, \kappa)=-L^{-1}\left[\sqrt{\frac{\pi}{t}} L\left[\bar{u}_{1}(y, \kappa)\right]\right]=(6-\kappa)\left[\frac{1}{2} \pi y^{2}+\frac{8}{15} \pi y^{5 / 2}\right] \\
\bar{u}_{3}(y, \kappa)=-L^{-1}\left[\sqrt{\frac{\pi}{t}} L\left[\bar{u}_{2}(y, \kappa)\right]\right]=-(6-\kappa)\left[\frac{8}{15} \pi y^{5 / 2}+\frac{1}{6} \pi^{2} y^{3}\right] \\
\vdots
\end{array}\right.
$$

then putting all the values of Eq. (35) termwise on the right-hand side into Eq. (33) and canceling all the noise terms, we get

$$
\bar{\psi}(y, \kappa)=(6-\kappa) y
$$

thus the parametric solution of Eq. (26) is

$$
(\underline{\psi}(y, \kappa), \bar{\psi}(y, \kappa)=((4+\kappa) y,(6-\kappa) y),
$$

which is the exact solution. Hence the solution is in close agreement with the solution computed in [34] by HPM and modified HPM.

Example 3 ([43]) Considered the fuzzy singular Volterra integral equation with Abel's type kernel

$$
\psi(y, \kappa)=(2 \sqrt{y}(\kappa-1), 2 \sqrt{y}(1-\kappa))-\int_{0}^{y} \frac{\psi(t, \kappa), \bar{\psi}(t, \kappa)}{\sqrt{y-t}} d t
$$

whose exact solution is $\left(\left(1-(\exp )^{\pi y} \operatorname{erfc} \sqrt{\pi y}\right)(\kappa-1),\left(1-(\exp )^{\pi y} \operatorname{erfc} \sqrt{\pi y}\right)(1-\kappa)\right)$.

The parametric form of Eq. (36) is written as

$$
\left\{\begin{array}{l}
\underline{\psi}(y, \kappa)=2 \sqrt{y}(\kappa-1)-\int_{0}^{y} \frac{\frac{\psi(t, \kappa)}{\sqrt{y-t}} d t}{\bar{\psi}(y, \kappa)=2 \sqrt{y}(1-\kappa))-\int_{0}^{y} \frac{\frac{\psi}{\psi(t, \kappa)}}{\sqrt{y-t}} d t} .
\end{array}\right.
$$

For the lower limit solution, taking the lower limit equation of Eq. (37) and applying Laplace transform, Theorem 1, and the inverse Laplace transform, we obtain

$$
\underline{\psi}(y, \kappa)=2 \sqrt{y}(\kappa-1)-L^{-1}\left[\sqrt{\frac{\pi}{t}} L[\underline{\psi}(y, \kappa)] .\right.
$$

Let the solution of Eq. (38) be in the form of an infinite series as

$$
\underline{\psi}(y, \kappa)=\sum_{j=0}^{\infty} \underline{u}_{j}(y, \kappa)
$$

Putting Eq. (39) into Eq. (38), we get

$$
\sum_{j=0}^{\infty} \underline{u}_{j}(y, \kappa)=2 \sqrt{y}(\kappa-1)-L^{-1}\left[\sqrt{\frac{\pi}{t}} L\left[\sum_{j=0}^{\infty} \psi(y, \kappa)\right]\right] .
$$


Now comparing Eq. (40) termwise and solving, we obtain

$$
\left\{\begin{array}{l}
\underline{u}_{0}(y, \kappa)=2 \sqrt{y}(\kappa-1), \\
\underline{u}_{1}(y, \kappa)=-L^{-1}\left[\sqrt{\frac{\pi}{t}} L\left[\underline{u}_{0}(y, \kappa)\right]\right]=-\pi y(\kappa-1), \\
\underline{u}_{2}(y, \kappa)=-L^{-1}\left[\sqrt{\frac{\pi}{t}} L\left[\underline{u}_{1}(y, \kappa)\right]\right]=\frac{4}{3} \pi y^{3 / 2}(\kappa-1), \\
\underline{u}_{3}(y, \kappa)=-L^{-1}\left[\sqrt{\frac{\pi}{t}} L\left[\underline{u}_{2}(y, \kappa)\right]\right]=-\frac{1}{2} \pi^{2} y^{2}(\kappa-1), \\
\vdots
\end{array}\right.
$$

then putting Eq. (41) into Eq. (39) on right-hand side and simplifying, as well as using the Mittag-Leffler function defined in Eq. (4), we get

$$
\begin{aligned}
\underline{\psi}(y, \kappa) & =(\kappa-1) \frac{\sum_{j=0}^{\infty}(-1)^{j-1}\left(\pi^{3} y\right)^{\frac{j}{2}}}{\Gamma(j / 2+1)} \\
& =1-E_{1 / 2}(-\sqrt{\pi y}(\kappa-1)) \\
& =\left(1-(\exp )^{\pi y} \operatorname{erfc} \sqrt{\pi y}\right)(\kappa-1) .
\end{aligned}
$$

Now for the upper limit solution, taking the upper limit equation of Eq. (36), we have

$$
\bar{\psi}(y, \kappa)=2 \sqrt{y}(1-\kappa)-L^{-1}\left[\sqrt{\frac{\pi}{t}} L[\bar{\psi}(y, \kappa)]\right] .
$$

Let the solution of Eq. (42) be in the form of an infinite series as

$$
\underline{\psi}(y, \kappa)=\sum_{j=0}^{\infty} \underline{u}_{j}(y, \kappa)
$$

Putting Eq. (43) into Eq. (42), we get

$$
\sum_{j=0}^{\infty} \bar{\psi}_{j}(y, \kappa)=2 \sqrt{y}(1-\kappa)-L^{-1}\left[\sqrt{\frac{\pi}{t}} L\left[\sum_{j=0}^{\infty} \bar{\psi}_{j}(y, \kappa)\right]\right] .
$$

Comparing Eq. (44) termwise and simplifying, we obtain

$$
\left\{\begin{array}{l}
\bar{u}_{0}(y, \kappa)=2 \sqrt{y}(1-\kappa), \\
\bar{u}_{1}(y, \kappa)=-L^{-1}\left[\sqrt{\frac{\pi}{t}} L\left[\bar{u}_{0}(y, \kappa)\right]\right]=-\pi y(1-\kappa), \\
\bar{u}_{2}(y, \kappa)=-L^{-1}\left[\sqrt{\frac{\pi}{t}} L\left[\bar{u}_{1}(y, \kappa)\right]\right]=\frac{4}{3} \pi y^{3 / 2}(1-\kappa), \\
\bar{u}_{3}(y, \kappa)=-L^{-1}\left[\sqrt{\frac{\pi}{t}} L\left[\bar{u}_{2}(y, \kappa)\right]\right]=-\frac{1}{2} \pi^{2} y^{2}(1-\kappa), \\
\vdots
\end{array}\right.
$$

then putting all the values of Eq. (45) termwise into Eq. (43) into the right-hand side and simplifying, using the Mittag-Leffler function defined in Eq. (4), we obtain

$$
\bar{\psi}(y, \kappa)=(1-\kappa) \frac{\sum_{j=0}^{\infty}(-1)^{j-1}\left(\pi^{3} y\right)^{\frac{j}{2}}}{\Gamma(j / 2+1)}
$$




$$
\begin{aligned}
& =1-E_{1 / 2}(-\sqrt{\pi y})(1-\kappa) \\
& =1-\left((\exp )^{\pi y} \operatorname{erfc} \sqrt{\pi y}\right)(1-\kappa) .
\end{aligned}
$$

Thus the parametric solution of Eq. (36) is given by

$$
(\underline{\psi}(y, \kappa), \bar{\psi}(y, \kappa))=\left(\left(1-(\exp )^{\pi y} \operatorname{erfc} \sqrt{\pi y}\right)(\kappa-1),\left(1-(\exp )^{\pi y} \operatorname{erfc} \sqrt{\pi y}\right)(1-\kappa)\right) .
$$

Clearly, the solution is the same as computed by HPM in [34].

\section{Conclusion}

In the present research, the main purpose is to establish an efficient algorithm for solving fuzzy convolution Volterra integral equation with Abel's type kernel by applying the LADM. The proposed algorithm is a combination of the Laplace transformation and the ADM. First, we apply the Laplace transformation and then, by expanding the unknown function in a series, we find the solution of the given problem. Further the efficiency, simplicity, and accuracy of the proposed algorithm are illustrated by giving some numerical examples which show the exactness of the proposed method. In the literature, the existing methods, like HPM, depend upon parameters and give approximate solutions while LADM is easier and more convenient. For a future work, we will investigate an efficient algorithm to solve both linear and nonlinear fuzzy Volterra integral equations and fuzzy Fredholm integral equation with separable type kernel using the LADM.

\section{Acknowledgements}

We are thankful to the reviewers for their careful reading and constructive suggestions which improved this paper considerably.

\section{Funding}

The first author was supported financially while writing this article from her own sources.

\section{Abbreviations}

ADM,Adomian Decomposition Method; LADM,Laplace Adomian Decomposition Method; HPM,Homotopy Perturbation Method.

\section{Availability of data and materials}

Data sharing not applicable to this article.

\section{Competing interests}

The authors have no conflict of interest regarding this work.

Authors' contributions

The authors contributed equally and approved the final draff of this manuscript.

\section{Author details}

${ }^{1}$ Department of Basic Sciences, Princess Sumaya University for Technology, Amman, Jordan. ${ }^{2}$ Department of Mathematics, University of Malakand, Dir(L), Khyber Pakhtunkhwa, Pakistan.

\section{Publisher's Note}

Springer Nature remains neutral with regard to jurisdictional claims in published maps and institutional affiliations.

Received: 10 December 2019 Accepted: 7 April 2020 Published online: 15 April 2020

References

1. Zadeh, L.A.: Fuzzy sets. Inf. Control 8, 338-353 (1965)

2. Chang, S.S.L., Zadeh, L.A.: On fuzzy mapping and control. IEEE Trans. Syst. Man Cybern. 2, 30-34 (1972)

3. Dubois, D., Prade, H.: Towards fuzzy differential calculus. Fuzzy Sets Syst. 8, 1-7 (1982)

4. Goetschel, R., Vaxman, W.: Elementary fuzzy calculus. Fuzzy Sets Syst. 18, 31-43 (1986)

5. Kaleva, O.: Fuzzy differential. Fuzzy Sets Syst. 24, 301-317 (1987) 
6. Dubois, D., Prade, H.: Towards fuzzy differential calculus part 1: integration of fuzzy mappings. J. Approx. Theory 8(1), $1-17$ (1982)

7. Sing, H., Gupta, M.M., Meitzler, T., Hou, Z.G., Garg, K.K., Solo, A.M.G., Zadeh, L.A.: Real-life application of fuzzy logic. Adv. Fuzzy Syst. 2013, Article ID 581879, 3 pages (2013)

8. Parihar, A.S., Verma, O.P., Khanna, C.: Fuzzy-contextual contrast enhancement. IEEE Trans. Image Process. 26, 1810-1819 (2017)

9. Matloka, M.: On fuzzy integrals. In: Proc. 2nd Polish Symp. on Interval and Fuzzy Mathematics Politechnika Poznansk, vol. 34, pp. 167-170 (1987)

10. Nanda, S.: On integration of fuzzy mappings. Fuzzy Sets Syst. 32(1), 95-101 (1989)

11. Jafarian, A., Nia, S.M.: Application of Taylor expansion method for the Volterra fuzzy integral equations system. Acta Univ. M. Belii Ser. Math. 20, 21-34 (2012)

12. Ghanbari, M., Allahviranloo, T: A new application of homotopy analysis method. Thai J. Math. 10(1), 43-57 (2012)

13. Matinfar, M., Saeidy, M.: Application of homotopy perturbation method for fuzzy integral equations. J. Math. Comput. Sci. 1(4), 377-385 (2010)

14. Ezzati, R., Ziari, S.: Numerical solution and error estimation of fuzzy Fredholm integral equation using fuzzy Bernstein polynomials. Aust. J. Basic Appl. Sci. 5(9), 2072-2082 (2011)

15. Ahmadian, A., Salahshour, S., Chan, C.S., Baleanu, D.: Numerical solutions of fuzzy differential equations by an efficient Runge-Kutta method with generalized differentiability. Fuzzy Sets Syst. 331, 47-67 (2018)

16. Dhandapani, P.B., Baleanu, D., Thippan, J., Sivakumar, V.: Fuzzy type RK4 solutions to fuzzy hybrid retarded delay differential equations. Front. Phys. 7(168), 1-6 (2019)

17. Slahshour, S., Ahmadian, A., Baleanu, D.: Variation of constant formula for the solution of interval differential equations of non-integer order. Eur. Phys. J. Spec. Top. 226, 3501-3512 (2017)

18. Ahmadian, A., Ismail, F., Salahshour, S., Baleanu, D., Ghaemi, F.: Uncertain viscoelastic models with fractional order: a new spectral tau method to study the numerical simulations of the solution. Commun. Nonlinear Sci. Numer. Simul. 53, 44-64 (2017)

19. Agarwala, R.P., Baleanu, D., Nieto, J.J., Torrese, D.F.M., Zhou, Y.: A survey on fuzzy fractional differential and optimal control nonlocal evolution equations. J. Comput. Appl. Math., 339, 3-29 (2018)

20. Otadi, M., Molish, M.: Numerical solution of fuzzy nonlinear integral equations of the second kind. Iran. J. Fuzzy Syst. $11,135-145(2014)$

21. Shafiee, M., Abbasbandy, S., Allahviranloo, T.: Predictor-corrector method for nonlinear fuzzy Volterra integral equations. Aust. J. Basic Appl. Sci. 5(12), 2865-2874 (2011)

22. Allahveranloo, T., Khezerloo, M., Sedaghatfar, O., Salahshour, S.: Toward the existence and uniqueness of solutions of second kind fuzzy Volterra integro-differential equations with fuzzy kernel. Neural Comput. Appl. 22(1), 133-141 (2013)

23. Fadravi, H.H., Buzhabadi, R., Nik, H.S.: Solving linear Fredholm fuzzy integral equations of the second kind by artificial neural networks. Alex. Eng. J. 53, 249-257 (2014)

24. Mirzaee, F:: Numerical solution of Fredholm fuzzy integral equations of the second kind using hybrid of block-pulse functions and Taylor series. Ain Shams Eng. J. 5, 631-636 (2014)

25. Hajighasemi, S., Allahveranloo, T., Khezerloo, M., Khorasany, M., Salahshour, S.: Existence and uniqueness of fuzzy Volterra integro-differential equations. Inf. Process. Manag. Uncertainty Knowl. Based Syst. 81, 491-500 (2010)

26. Agarwal, R.P., Lakshmikantham, V., Nieto, J.J: On the concept of solution for fractional differential equations with uncertainty. Nonlinear Anal. 72(6), 2859-2862 (2010)

27. El-Sayed, A.M.A.: Fractional-order diffusion-wave equation. Int. J. Theor. Phys. 35(2), 311-322 (1996)

28. Nieto, J.J.: Maximum principles for fractional differential equations derived from Mittag-Leffler functions. Appl. Math Lett. 23(10), 1248-1251 (2010)

29. Shen, Y., Yang, S., Sui, C.: Analysis on limit cycle of fractional-order van der Pol oscillator. Chaos Solitons Fractals 67 , 94-102 (2014)

30. Wazwaz, A.M.: Linear and Non-linear Integral Equations Methods and Applications. Higher Education Press, Beijing (2011)

31. Wazwaz, A.M.: A First Course in Integral Equations, 2nd edn. World Scientific, Singapore (2007)

32. Pandey, R.K., Singh, O.P., Singh, V.K.: Efficient algorithms to solve singular integral equations of Abel's type. Comput. Math. Appl. 57, 664-676 (2009)

33. Singh, V.K., Pandey, R.K., Singh, O.P.: New stable numerical solutions of singular integral equations of Abel's type by using normalized Bernstein polynomials. Appl. Math. Sci. 3, 241-255 (2009)

34. Herman, G.T.: Image reconstruction from projections. Real-Time Imaging 1, 3-18 (1995)

35. Liao, S.: Comparison between the homotopy analysis method and homotopy perturbation method. Appl. Math. Comput. 169, 1186-1194 (2005)

36. Shah, R., Khan, H., Arif, M., Kumam, P.: Application of Laplace Adomian decomposition method for the analytical solution of third-order dispersive fractional partial differential equations. Entropy 21, 335 (2019). https://doi.org/10.3390/e21040335

37. Manafianheris, J.: Solving the integro-differential equations using the modified Laplace Adomian decomposition method. J. Math. Ext. 6(1), 41-55 (2012)

38. Allahviranloo, T., Barkhordari Ahmadi, M.: Fuzzy Laplace transforms. Soft Comput. 14, 235-243 (2010)

39. Salahshour, S., Khezerloo, M., Hajighasemi, S., Khorasany, M.: Solving fuzzy integral equations of the second kind by Laplace transform method. Int. J. Ind. Math. 4(1), 21-29 (2012)

40. Li, Y., Chen, Y.Q., Podlubny, I.: Mittag-Leffler stability of fractional order nonlinear dynamic systems. Automatica 45(8), 1965-1969 (2009)

41. Friedman, M., Ma, M., Kandel, A.: Numerical solution of fuzzy differential and integral equations. Fuzzy Sets Syst. 106 35-48 (1999)

42. Park, J.Y., Jeong, J.U.: A note on fuzzy integral equations. Fuzzy Sets Syst. 108(2), 193-200 (1999)

43. Ullah, S., Farooq, M., Ahmad, L.: Efficient algorithm for solving fuzzy Abel singular integral equation using homotopy perturbation transform method. Bull. Malays. Math. Sci. Soc. 2, 1-18 (2014) 